\title{
Research on Depression, Anxiety and Memory of Subhealth after Practicing Jianshenqigong-Wuqinxi
}

\author{
Yan Wang ${ }^{1}$, Changle Chen ${ }^{2}$, Zhang Zhang ${ }^{3}$ \\ ${ }^{1}$ School of Medical Instrument and Food Engineering, University of Shanghai for Science and Technology, \\ Shanghai, China \\ ${ }^{2}$ Shanghai Qigong Institute, Shanghai, China \\ ${ }^{3}$ Dongzhimen Hospital Affiliated to Beijing University of Chinese Medicine, Beijing, China \\ E-mail: lunayan100@hotmail.com, chenchangle2002@sohu.com \\ Received May 26, 2010; revised July 27, 2010; accepted August 8, 2010
}

\begin{abstract}
Objective: To study the effect of practicing Jianshenqigong-wuqinxi on subhealth problems including depression, anxiety, and memory loss. Methods: 1) 80 cases with subhealth problem were randomly divided into two groups. 40 cases practicing Jianshenqigong-wuqinxi were used as test group, and the other 40 unpracticing cases were used as control group. 2) The test group was practising Jianshenqigong-wuqinxi one hour in the morning five times per week for three months. 3) Scores of subhealth status such as depression and anxiety were evaluated once every two weeks, while the memory test was performed once a month. 4) Data analysis: SPSS11.0 software was used for statistical analyses. Data were shown in mean \pm SEM. Difference was considered significant if the t-test P value is less than 0.05 . Results: 1 ) Scores of subhealth state and depression were significantly lower in test group than those of control group. The beneficial effects of practicing Jianshenqigong-wuqinxi were more obvious for the subjects in the test group who had practiced Jianshenqigong-wuqinxi for two to twelve weeks $(\mathrm{P}<0.05)$. 2) Scores of anxiety test were significantly lower in test group than those of control group after practicing Jianshenqigong-wuqinxi for six to twelve weeks $(\mathrm{P}<0.05)$. 3) Scores of memory were significantly higher in test group than those of control group $(\mathrm{P}<$ 0.05). Conclusions: 1) Practicing Jianshenqigong-wuqinxi could promote health in people who have subhealth mental problems. 2) Practicing Jianshenqigong-wuqinxi could relieve depression, anxiety while enhance memory.
\end{abstract}

Keywords: Subhealth, Depression, Anxiety, Jianshenqigong-Wuqinxi

\section{Introduction}

Subhealth is a kind of status between health and disease, which has no precise definition but is generally defined as low physical activity and adaptability regularly occur. It has been reported from WHO that there are more than 70 percent people in the world are in subhealth status $[1,2]$. In China the subhealthy people are about 60 to 70 percent, most of which are between 35 to 45 years old. The Chinese in subhealth conditions have exceeded 7 million according to recent data [3,4]. For those people they are often disturbed by bad feelings such as depression, anxiety, panic, short breath, listlessness, dizziness, headache, insomnia, etc. Jianshenqigong-wuqinxi mimics five animals' appearances and actions such as tiger, deer, bear, monkey, and crane. And it is a kind of mildly intense aerobic exercise, which makes vasodilatation, regulates autonomic nerve, increases venous return and enhances body sensibility. It was supposed that practicing Jianshenqigong-wuqinxi could relieve the bad feelings characteristic of subhealthy people. To discover the mechanism of physiological and psychological changes of Jianshenqigong wuqinxi on subheathy people, we observe the effects of practicing Jianshenqigong-wuqinxi on depression, anxiety, and memory loss of subhealthy people.

\section{Methods}

1) 80 cases (not hospitalized patients) with subhealth selected from Tianlin community and Muyang hospital were randomly divided into 2 groups. Subjects in the test 
group (40 cases) were practicing Jianshenqigong -wuqinxi, while subjects in the control group (40 cases) did not practice Jianshenqigong ·wuqinxi.

2) The test group was practicing Jianshenqigongwuqinxi one hour in the morning five times per week for three months.

3) Scores of subhealth staus such as depression and anxiety were evaluated once every two weeks, while the memory test was evaluated once a month.

4) Index of test:

a) The diagnosis of subhealth was according to the Fukuda complete text of revised case definition in American control disease center (CDC) in 1994 [5]. Nine items were tested in diagnosis of subhealth. The subjects should answer each item as yes or no. If the answer was yes, 1 score would be added (except the first item). Two scores would be added for the first item. After all the items were finished, the person whose score more than 6 was considered as in subhealth status.

b) Self-rating Depression Scale (SDS) and Self-Rating Anxiety (SAS) tables were used in depression and anxiety test accordingly. Twenty items were tested in calculation of scores of SDS and SAS. Each item has 4 grades. The first grade is 'no', the second is 'some time', the third is 'many time' and the forth is 'most or all the time'. SDS scores less than 40 were 'normal', between 40 and 60 were 'mild depress', more than 60 were 'heavy depress'. SAS scores less than 40 were 'normal' between 40 and 60 were 'mild anxiety' more than 60 were 'heavy anxiety'.

c) Memory test: a list of random numbers or letters was presented on a computer screen at the rate of one per two seconds. The subjects being tested were asked to recall the items in order, and if they are correct, the computer would record the length of the numbers or letters as the memory of the subjects. The test began with two numbers, increasing until the subjects committed twice errors. Recognizable patterns (for example 2, 4, 6, 8) should be avoided [6].

5) Data analysis: SPSS11.0 software was used. Data were shown in mean \pm SEM. Difference was considered significant at $\mathrm{P}<0.05$.

\section{Rerults}

1) Scores of subhealthy state (Table 1) were significantly lower than that of control group. The beneficial effects of practicing Jianshenqigong-wuqinxi were more obvious for the subjects in the test group who had practiced Jianshenqigong-wuqinxi for two to twelve weeks $(\mathrm{P}<0.05)$. There were also significant difference of subhealth scores in control group before and after two to twelve weeks $(\mathrm{P}<0.05)$.

2) Scores of anxiety (Table 2) of test group were sig-
Table 1. Scores of subhealth after practicing Jianshenqigong-wuqinxi for twelve weeks.

\begin{tabular}{|c|c|c|}
\hline Time ( weeks ) & Control group & Test group \\
\hline 0 & $6.45 \pm 0.36$ & $6.40 \pm 0.40$ \\
\hline 2 & $6.1 \pm 0.7^{\text {年 }}$ & $5.20 \pm 1.45^{\text {牥 }}$ \\
\hline 4 & $6.18 \pm 0.76^{\text {光 }}$ & $4.95 \pm 1.74$ \\
\hline 6 & $6.03 \pm 1^{\text {药 }}$ & $4.65 \pm 1.52^{\text {部 }}$ \\
\hline 8 & $5.88 \pm 1.19^{\text {病 }}$ & $4.53 \pm 1.54$ \\
\hline 10 & $5.75 \pm 1.16^{\text {六 }}$ & $4.38 \pm 1.24$ \\
\hline 12 & $5.78 \pm 1.48^{\text {疗 }}$ & $4.5 \pm 1.51^{\text {名 } \Delta}$ \\
\hline
\end{tabular}

There was significant difference when compared with before practicing $(\mathrm{P}<0.05)$; ${ }^{\triangle}$ There was significant difference when compared with control group $(\mathrm{P}<0.05)$.

Table 2. Anxiety scores of subhealth after practicing Jianshenqigong-wuqinxi for twelve weeks.

\begin{tabular}{|c|c|c|}
\hline Time（weeks ) & Control group & Test group \\
\hline 0 & $36.8 \pm 7.86$ & $36.8 \pm 7.38$ \\
\hline 2 & $36.5 \pm 6.74$ & $35.5 \pm 7.91$ \\
\hline 4 & $36 \pm 8.12$ & $34.7 \pm 7.14$ \\
\hline 6 & $35.8 \pm 8.08$ & $32.5 \pm 6.77^{\text {紊 }}$ \\
\hline 8 & $36.0 \pm 8.12$ & $32.1 \pm 7.55^{\text {玆 }}$ \\
\hline 10 & $35.5 \pm 8.31$ & $32.4 \pm 7.79$ \\
\hline 12 & $35.6 \pm 8.52$ & $31.9 \pm 7.52^{\text {勏 }}$ \\
\hline
\end{tabular}

There was significant difference when compared with before practicing ( $\mathrm{P}<0.05) ;{ }^{\triangle}$ There was significant difference when compared with control group $(\mathrm{P}<0.05)$.

nificantly lower than that of control group. The beneficial effects of practicing Jianshenqigong-wuqinxi were more obvious for the subjects in the test group who had practiced Jianshenqigong-wuqinxi for six to twelve weeks $(\mathrm{P}<0.05)$. There was no significant difference of anxiety scores in control group before and after two to twelve weeks $(\mathrm{P}>0.05)$.

3) Scores of depression (Table 3) of test group were significantly lower than that of control group. The beneficial effects of practicing Jianshenqigong-wuqinxi were more obvious for the subjects in the test group who had practiced Jianshenqigong-wuqinxi for two to twelve weeks $(\mathrm{P}<0.05)$. There was no significant difference of anxiety scores in control group before and after two to twelve weeks $(\mathrm{P}>0.05)$.

4) Scores of memory (Table 4) were higher in test group than that of control group. The beneficial effects of practicing Jianshenqigong-wuqinxi were more obvious for the subjects in the test group who had practiced Jianshenqigong-wuqinxi for twelve weeks $(\mathrm{P}<0.05)$. There was no significant difference of memory scores in control group before and after two to twelve weeks ( $\mathrm{P}>$ $0.05)$. 
Table 3. Depression scores of subhealth after practicing Jianshenqigong-wuqinxi for twelve weeks.

\begin{tabular}{|c|c|c|}
\hline Time ( weeks ) & Control group & Test group \\
\hline 0 & $40.68 \pm 9.12$ & $40.40 \pm 8.86$ \\
\hline 2 & $39.90 \pm 9.88$ & $36.08 \pm 9.47^{\sqrt{2} \Delta}$ \\
\hline 4 & $39.85 \pm 9.98$ & $35.78 \pm 9.52^{\text {后 } \Delta}$ \\
\hline 6 & $39.53 \pm 8.86$ & $35.73 \pm 8.79^{\text {社 }}$ \\
\hline 8 & $39.38 \pm 8.88$ & $35.60 \pm 8.90^{\sqrt{2} \Delta}$ \\
\hline 10 & $38.92 \pm 7.92$ & $35.06 \pm 9.15^{\text {斻 }}$ \\
\hline 12 & $38.64 \pm 8.61$ & $35.34 \pm 8.46^{\grave{\lambda} \Delta}$ \\
\hline
\end{tabular}

There was significant difference when compared with before practicing $(\mathrm{P}<0.05) ;{ }^{\triangle}$ There was significant difference when compared with control group $(\mathrm{P}<0.05)$.

Table 4. Memory (digits) test of subhealth after practicing Jianshenqigong-wuqinxi for twelve weeks.

\begin{tabular}{ccc}
\hline Time ( weeks ) & Control group & Test group \\
\hline 0 & $7.28 \pm 2.53$ & $7.13 \pm 1.94$ \\
4 & $7.28 \pm 2.53$ & $7.63 \pm 1.92$ \\
8 & $7.18 \pm 2.23$ & $8.2 \pm 2.61$ \\
12 & $7.17 \pm 2.47$ & $8.86 \pm 2.00^{\text {衫 }}$
\end{tabular}

There was significant difference when compared with before practicing $(\mathrm{P}<0.05) ;{ }^{\triangle}$ There was significant difference when compared with control group $(\mathrm{P}<0.05)$.

\section{Conclusions}

1) Practicing Jianshenqigong-wuqinxi could promote health in people who have subhealth mental problems.

2) Practicing Jianshenqigong wuqinxi could relieve bad feelings such as depression, anxiety of subhealth people and enhance memory.

\section{Discussions}

Many researchers have observed the effect of practicing Jianshenqigong-wuqinxi on different people. Jing-mei $\mathrm{Wu}$ found after 3-month practicing, Jianshenqigongwuqinxi could improve the immunity of the people, and the immunity ability of woman practitioners grew even faster [7]. Ding-hai Yu found after 6-month exercise of Jianshenqigong-wuqinxi, the NK cell activity of exercisers in the experimental group was higher than that before exercise and control group [8]. But no scientific work studied the effect of Jianshenqigong-wuqinxi on subhealth people and no people observed the physiological and psychological changes of practicers. We discovered the effects of practicing Jianshenqigong-wuqinxi on subhealth mental problems. Subhealth status is characterized by panic, short breath, dizziness, headache, insomnia, etc.

Subhealth is the major cause of many diseases [9].
People with subhealth problems could become severely ill later in their lifetime, which were highly correlated with their subhealth problems [10]. Therefore optimal intervention of subhealth problems is necessary. In our experiment we found that there were significant difference of subhealth scores between Jianshenqigongwuqinxi practitioners and controls (Table 1), which suggested Jianshenqigong-wuqinxi could promote mental health of people who have subhealth problems. Comparison with controls, more cases of subhealthy practicers became mentally healthy after practicing Jianshenqigong-wuqinxi for 3 months, suggesting the beneficial effects of Jianshenqigong-wuqinxi.

It was reported that tai chi could regulate psychological health of subhealthy people, and relieve bad feelings such as lassitude, memory loss, irritable, anxiety, insomnia, etc [11]. Jianshenqigong-wuqinxi is a kind of mildly intense aerobic exercise just like tai chi. From our experiment we found practicing Jianshenqigong-wuqinxi could relieve bad feelings such as anxiety (Table 2) and depression (Table 3), while enhance memory (Table 4). The reasons might be as follows: a) Jianshenqigongwuqinxi is a kind of exercise that can regulate bad feelings such as anxiety and depression through releasing of biological macromolecules such as adrenocorticotropic hormone, cortisol, catecholamine, opioid peptides, etc. These molecules are believed to be beneficial to psychological status [12,13]. b) Jianshenqigong-wuqinxi has multiple mental stages. Psychological self-regulating was required for practicing each stage of Jianshenqigongwuqinxi. For example, when practicing the stage of 'tiger-xi', the practitioners will mimic a powerful tiger. When practicing the stage of 'deer-xi', the practitioners will mimic a gentle deer. Such changes of psychological stages were reported to be beneficial to reducing bad feelings [14].

It is therefore concluded that the effects of practicing Jianshenqigong-wuqinxi have significant beneficial effects on improving the overall mental health of people suffering from subhealth problems.

\section{Acknowledgements}

This work was supported by grants awarded to Chen Chang-le from the General Administration of Sport of China (QG200093), and to Yan Wang from Science and Technology Commission of Shanghai Municipality (Outstanding Youth Foundation of University of Shanghai for Science and Technology).

\section{References}

[1] L. D. Wang, "Chronic Disease and Subhealth in China: 
The Effect and the Guideline of Prevention and Treatment," Chinese Medical Journal, Vol. 83, No. 12, June 2003, pp.1031-1034.

[2] X. L. Wang, Y. H. Huo, J. Li, X. S. Zhao and R. Luo, "Evaluation of the Questionnaire for Subhealth Status Survey Based on the Symptoms in Traditional Chinese Medicine," Journal of Southern Medical University, Vol. 27, No. 2, February 2007, pp. 160-163.

[3] T. F. Wang, J. J. Wang, X. L. Xue, P. Han, Y. J. Zhang, G. R. Li, X. Y. Wu, Y. Zhao, L. L. Tang, Y. Y. Liu, C. L. Sui, C. Fu, Y. M. Shang and B. Zhou, "Distribution Characteristics of Traditional Chinese Medicine Syndromes and their Elements in People with Subhealth Fatigue," Journal of Chinese Integrative Medicine, Vol. 8, No. 3, March 2010, pp. 220-223.

[4] Y. H. Huo, X. L. Wang, J. Li, C. Y. Zhu, X. M. Sun, X. S. Zhao, C. Zhang and R. Luo, "Analysis of Clinical Manifestations of Subheath Status in a Medical College of Guangdong Province,” Journal of Southern Medical University, Vol. 27, No. 4, April 2007, pp. 448-449.

[5] K. Fukuda, S. E. Straus, I. Hickie, M. C. Sharpe, J. G. Dobbins and A. Komaroff, "The Chronic Fatigue Syndrome: A Comprehensive Approach to its Definition and Study. International Chronic Fatigue Syndrome Study Group,” Annals of Internal Medicine, Vol. 121, No. 12, December 1994, pp. 953-959.

[6] G. Miller, "The Magical Number Seven, Plus or Minus Two: Some Limits on Our Capacity for Processing Information,” Psychological Review, Vol. 101, No. 2, April 1994, pp. 343-352.

[7] J. M. Wu and D. H. Yu "Research on Effects of Practicing 'Wuqinxi' on the Outer Sub-Group of T-Cell of the Middle-Aged and Old People," Journal of Beijing Sport
University, Vol. 29, No. 8, August 2006, pp. 1074-1076.

[8] D. H. Yu and J. M. Wu, "Effects of Exercising Building-Up Qigong-Wuqinxi on Middle-Aged and Old People's NK Cell Activity,” Journal of Shanghai University of Sport, Vol. 32, No. 1, January 2008, pp. 56-59.

[9] H. W. Jiang, "Integrative Medical Research Thinking on the Constitution-Subhealth-Disease Relation Developing Axis," Chinese Journal of Integrated Traditional and Western Medicine, Vol. 29, No. 9, September 2009, pp. 836-837.

[10] C. X. Fan, S. Y. Wang, L. Zhu, Y. J. Xiao and S. B. Ma, "Study on the Prevalence and Risk Factors of 'Subhealth' Status in College and University Staff in Guangdong Province,” Zhonghua Liu Xing Bing Xue Za Zhi, Vol. 24, No. 9, September 2003, pp. 774-777.

[11] H. Yin and J. Yang, "Rehabilitative Effects of Taichi Exercise on the Subhealthy Status of College Students," Journal of Clinical Rehabilitative Tissue Engineering Research, Vol. 39, No. 11, 2007, pp. 7991-7992.

[12] M. A. Nies and C. L. Motyka, "Factors Contributing to Women's Ability to Maintain a Walking Program," Journal of Holistic Nursing, Vol. 24, No. 1, March 2006, pp. 7-14.

[13] B. B. Simen, C. H. Duman, A. A. Simen and R. S. Duman, “TNFalpha Signaling in Depression and Anxiety: Behavioral Consequences of Individual Receptor Targeting,” Biological Psychiatry, Vol. 59, No. 9, February 2006, pp. 775-785.

[14] A.-H. Peng and Z. Yan, “Analysis of Epidemiological Status and Countermeasures for Sub-Health Problem among Occupational Poputation in China,” Modern Preventive Medicine, Vol. 36, No. 14, November 2009, pp. 2617-2619. 